\title{
Side-specific reward memories in honeybees
}

\author{
Mariana Gil, ${ }^{1}$ Randolf Menzel, and Rodrigo J. De Marco \\ Department of Biology, Chemistry, and Pharmacy, Institute of Biology and Neurobiology, Free University of Berlin, \\ 14195 Berlin, Germany
}

\begin{abstract}
We report a hitherto unknown form of side-specific learning in honeybees. We trained bees individually by coupling gustatory and mechanical stimulation of each antenna with either increasing or decreasing volumes of sucrose solution offered to the animal's proboscis along successive learning trials. Next, we examined their proboscis extension response (PER) after stimulation of each antenna 1, 2, 3, and $24 \mathrm{~h}$ after training. The bees extended their proboscises earlier after stimulation of the antenna that had been coupled with increasing volumes than after stimulation of the antenna that had been coupled with decreasing volumes, thereby revealing short- and long-term side differences in the bees' PE reaction time. The bees' reaction time correlated well with the reaction time of the muscles M17. Long-term side differences in reaction time were prevented by repetitive antennal stimulation. Mechanosensory input was indispensable and sufficient for revealing side differences in reaction time. Such differences were specific to the gustatory input that the bees experienced during training. Our results show that side differences in the bees' PE reaction time depend upon the activation of side-specific reward memories. These memories are formed via the combined effect of a specific property of reward, i.e., that its magnitude increases or decreases over time, and side information seemingly relying on mechanosensory input. We present a learning procedure suitable to study reward learning in honeybees, which includes precise behavioral measures, physiological correlates of behavior, and within-animal controls. This procedure will prove fruitful in pharmacological and electrophysiological analyses of the neural substrates underlying reward memories in honeybees.
\end{abstract}

[Supplemental material is available online at www.learnmem.org.]

Honeybees extend their proboscises reflexively when the gustatory receptors of their antennae, proboscis, and tarsi are stimulated with sucrose (Kuwabara 1957). This behavior allows them to gather sucrose solution, which constitutes their primary source of energy and acts as a sugar reward in appetitive learning (Takeda 1961). Because it is an innate behavior which can be calibrated through learning, the honeybees' proboscis extension response (PER) led to a well-established laboratory procedure for the study of learning and memory phenomena (Takeda 1961; Bitterman et al. 1983; Rehder 1987; Smith and Menzel 1989; Haupt 2004). Recently, we found that bees that had been presented with increasing volumes of sugar solution across successive learning trials extended their proboscises earlier in delayed tests, in comparison to bees that had been presented with either decreasing or constant volumes of sugar solution (Gil et al. 2008). It follows that harnessed bees learn that reward magnitude increases over time and adjust their PERs accordingly. Also, learning phenomena limited to input from one side of the sensory system, i.e., sidespecific learning, is well documented in honeybees. Honeybees learn to associate side-specific olfactory and mechanical stimulation of their antennae with sugar reward (Macmillan and Mercer 1987; Sandoz and Menzel 2001; Giurfa and Malun 2004). Habituation and sensitization can also be side specific (Braun and Bicker 1992; Sandoz et al. 2002). Thus, the wonder arises as to whether honeybees learn side specifically that reward magnitude increases or decreases over time.

Here, we asked whether honeybees associate the stimulation of each of their antenna with either increasing or decreasing volumes of sugar solution so as to subsequently adjust their PERs, depending on which antenna is stimulated. To answer this question, we developed a side-specific training procedure in which

'Corresponding author.

E-mail mgdm04@yahoo.com.ar; fax 49-030-83855455.

Article is online at http://www.learnmem.org/cgi/doi/10.1101/lm.1419109. gustatory and mechanical stimulation of each of a honeybee's antenna is coupled with either increasing or decreasing volumes of sugar solution offered to the animal's proboscis throughout a series of consecutive training trials. Using side-specificity, we incorporated within-individual controls into a behavioral procedure, which proved suitable for the analysis of behavioral correlates of memories of specific reward properties (Gil et al. 2008). By means of such a procedure, we asked a number of additional questions: Does such an association lead to short- and long-term memories? How are these side-specific reward memories extinguished? How do they develop during training? Can they be mapped to a physiological measure of behavior? What is the role of mechanical stimulation of the antennae in the formation of these side-specific memories? Are the underlying associations specific with respect to the gustatory input? What is the interplay between mechanical and gustatory inputs in the formation and retrieval of these memories? Our research contributes to the understanding of how honeybees learn specific properties of reward, and of how they learn and process side-specific stimuli.

\section{Results}

\section{Side-specific adjustments of a bee's PE reaction time}

We first asked whether honeybees learn side-specifically that the volume of sugar solution increases or decreases over time. We used a side-specific training in which the stimulation of each of a honeybee's antennae was coupled with either increasing or decreasing volumes of sucrose solution offered to the animal's proboscis throughout six consecutive training trials. Next, we tested the bees by stimulating each antenna separately. During both training and testing, we recorded the bees' PE reaction time after stimulation of each antenna (see Materials and Methods). When tested 1,2 , and $3 \mathrm{~h}$ after training, the bees showed a shorter $P E$ reaction time after stimulation of the antenna that had been 
coupled with increasing reward volumes than after stimulation of the antenna that had been coupled with decreasing reward volumes (Fig. 1A-C). The differential reaction time (i.e., the normalized difference between the reaction times of the decreasing and the increasing antennae) was $29.9 \%( \pm 6.78), 19.3 \%$ $( \pm 5.81)$, and $21.0 \%( \pm 6.51)$ in tests 1,2 , and $3 \mathrm{~h}$, respectively. These values were significantly higher than $0 \%$, i.e., the value that is expected if the reaction times were to be the same for both the decreasing and the increasing antennae (one sample $t$-test: $t_{(63)}=$ 4.39, $P<0.0001, t_{(65)}=3.32, P=0.001, t_{(71)}=3.22, P=0.002$, respectively). The same bees did not exhibit such side differences when tested $24 \mathrm{~h}$ after training (Fig. 1D). The corresponding differential reaction time was $9.31 \%( \pm 9.75)$, a value which did not differ from $0 \%$ (one sample $t$-test: $t_{(41)}=0.95, P=0.34$ ). By contrast, the bees that were tested only once $24 \mathrm{~h}$ after training (test $24 \mathrm{~h}_{\text {(we) }}$ [where "we" stands for "without extinction trials"]). did show side-specific reaction times (Fig. 1E), and the corresponding differential reaction time was $18.0 \%( \pm 8.0)$, a value that was significantly higher than $0 \%$ (one sample $t$-test: $t_{(45)}=2.24, P=$ $0.03)$. Thus, in the tests the trained bees extended their proboscis earlier after stimulation of the increasing antenna than after stimulation of the decreasing antenna. These side-specific adjustments of the PE reaction time can be observed 1, 2, 3, and $24 \mathrm{~h}$ after training and are, in the long term ( $24 \mathrm{~h}$ after training), prevented by repetitive antennal stimulation.
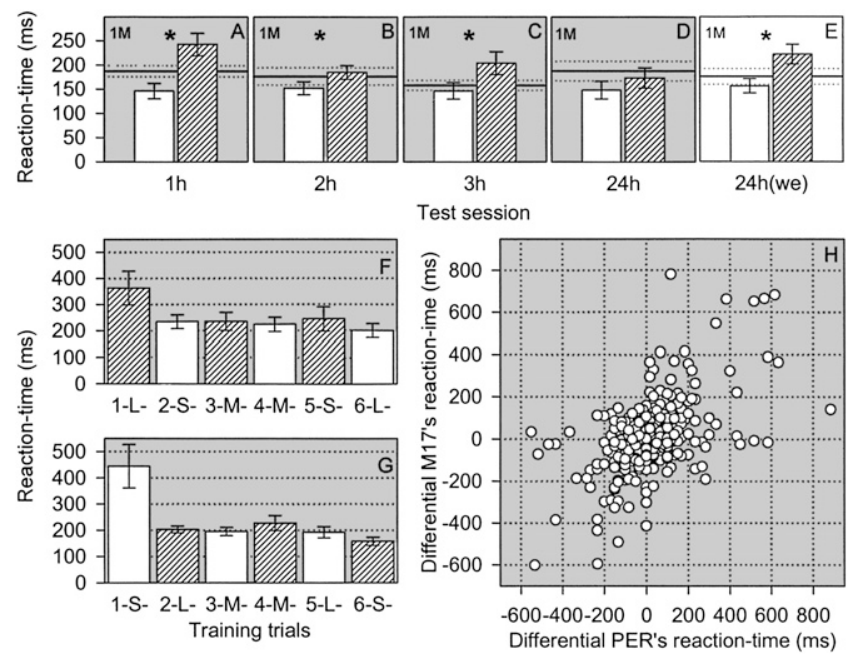

Figure 1. PE reaction times (mean $\pm S E M$, in milliseconds) following stimulation of the increasing and the decreasing antennae (white and dashed bars, respectively). Data are shown for both testing $(A-E)$ and training $(F, G)$. Bees were trained and tested with $1 \mathrm{M}$ sucrose solution. In $A-E$, asterisks indicate statistical differences (Wilcoxon signed-rank test) between the reaction times following stimulation of the increasing and decreasing antennae: $(A) W=-1168, P<0.0001, n=64 ;(B) W=-760$, $P=0.009, n=67 ;(C) W=-994, P=0.002, n=72 ;(D) W=-155, P=0.3$, $n=42 ;$ and $(E) W=-543, P=0.001, n=46$. Reference lines correspond to the PE reaction times of untrained bees tested simultaneously (mean \pm SEM, solid and dotted lines, respectively). $(F, G)$ Side-specific training consisted of coupling stimulation of each antenna with either increasing or decreasing volumes of sugar solution. It could start either with the smallest $(F, n=52)$ or the largest volume $(G, n=59)$. S, L, and M indicate the small, medium, and large volume of sugar solution, respectively. PE reaction times during training were analyzed by means of two-way repeated-measures ANOVA, with repeated measures on one factor, the training trials; the other being the type of training, i.e., starting with the small or the large reward volume: effects vs. $L, F_{(1,109)}=0.05, P=0.8$; effect training trials, $F_{(5,545)}=13.4, P<0.0001$; and effect ${ }_{\text {interaction, }} F_{(5,545)}=$ 1.24, $P=0.3$. $(H)$ Relationship between PE and M17 differential reaction times; each point represents an individual difference between the reaction time of the decreasing and the increasing antennae (see Materials and Methods).
We also asked whether the bees' PE reaction time changed during training. We did not find side-specific changes throughout training, although the bees' $\mathrm{PE}$ reaction time did change across the consecutive training trials (Fig. 1G,F). The PE reaction time in the first trial was higher than those of the remaining trials (Tukey's multiple comparisons, $P<0.0001$ in all cases). In addition, the PE reaction time in the second trial was higher than that of the last trial (Tukey's multiple comparisons, $P=0.005$ ). We found no differences between those of the remaining trials (Tukey's multiple comparisons, $P>0.05$ in all cases). Thus, the bees' $\mathrm{PE}$ reaction times diminished by $55 \%$ during training, and side-specific differences in PE reaction time were not observed. Moreover, we did not find differences while comparing the data from the bees whose training started with either the small or the large volume of sugar reward (Fig. 1F,G, respectively).

We also examined whether the side-specific differences in PE reaction time arose from either a reduction in the reaction time of the increasing antenna or, instead, an increase in the reaction time of the decreasing antenna, or both. To this end, we compared the PE reaction times of both trained and untrained bees. Untrained bees were fed with the same amount of sucrose solution offered to the trained bees, and their reaction time was recorded 1, 2, 3, 24, and 24 $\mathrm{h}_{\text {(we) }}$ after feeding (see Materials and Methods; Supplemental Fig. S1). In the first test, the mean PE reaction time of the untrained bees $(187.14 \pm 11.58 \mathrm{~ms}$; see reference lines in Fig. 1A) was higher than that of the trained bees following stimulation of their increasing antenna. It was also lower than that of the trained bees following stimulation of their decreasing antenna (Fig. 1A; Mann-Whitney test: $\left.P_{\text {ut vs. I }}=0.005, P_{\text {ut vs. D }}=0.04\right)$. In the tests performed 2,3 , and $24 \mathrm{~h}$ after training, the PE reaction times of the untrained and the trained bees did not differ from each other (see reference lines in Fig. 1B-D, respectively, $176.54 \pm 17.92,158.04 \pm 10.18,187.65 \pm$ $20.23 \mathrm{~ms}$; Mann-Whitney test: test $2 \mathrm{~h}, P_{\text {ut vs. I }}=0.4, P_{\text {ut vs. D }}=0.2$; test $3 \mathrm{~h}, P_{\text {ut vs. I }}=0.1, P_{\text {ut vs. D }}=0.2$; and test $24 \mathrm{~h} P_{\text {ut vs. I }}=0.1, P_{\text {ut vs. D }}$ $=0.5$ ). In the test $24 \mathrm{~h}_{\text {(we), }}$, the PE reaction time of the untrained bees was lower than that of the trained bees following stimulation of their decreasing antenna (see reference lines in Fig. 1E; $187.65 \pm$ $20.23 \mathrm{~ms}$; Mann-Whitney test: $\left.P_{\text {ut vs. I }}=0.5, P_{\text {ut vs. } \mathrm{D}}=0.04\right)$. Thus, side-specific differences in $\mathrm{PE}$ reaction time of the trained bees observed in the test performed $1 \mathrm{~h}$ after training arose from both a reduction and an increase in the reaction times that followed the stimulation of the increasing and the decreasing antennae, respectively (Fig. 1A). In the test $24 \mathrm{~h}_{\text {(we), }}$ such side-specific responses arose from an increase in the reaction time associated with the stimulation of the bees' decreasing antenna (Fig. 1E).

In parallel to the video recordings, we measured the activity of the muscles M17, which are a pair of bilaterally symmetrical muscles involved in the extension of the proboscis (Snodgrass 1956) (see Materials and Methods). We found that the PE reaction time correlated well with both the ipsi- and contralateral M17s reaction times (Spearman correlation: ipsi- $r=0.43, P<0.0001, n=$ 1028 and contra- $r=0.42, P<0.0001, n=1013)$. It also correlated with the average of the ipsi- and contralateral M17s reaction times (Spearman correlation: $r=0.44, P<0.0001$, and $n=1013$ ). Thus, we found a positive correlation between the PEs and M17s differential reaction times (Fig. 1H; Spearman correlation: $r=0.51, P<0.0001$, and $n=327$ ). The broad distribution of the points in Figure $1 \mathrm{H}$ is consistent with the well-known fact that the M17 muscles are not the only muscles responsible for a honeybee's PER.

\section{The role of mechanosensory input}

A honeybee's antenna not only has gustatory receptors, but also mechanoreceptors located in each of its two joints, i.e., the proximal joint between the head and the first antennal segment (scape), and the distal joint between the scape and the second antennal segment (flagellum) (Schneider 1964; Markl 1971). It 
follows that stimulation of a honeybee's antennae, in instances as in those of our experiments, involves inputs from gustatory as well as from mechanosensory receptors. We aimed to examine the roles of gustatory receptors located alongside the flagellum and of mechanoreceptors located between the scapes and the head in the development of side differences in PE reaction time. Thus, we hampered mechanical inputs from the joint between the head and the scape by fixing a honeybee's scapes to the head, so that the animal could move only the flagellum of each antenna (see Materials and Methods). Next, we examined the behavior of bees trained and tested with immobilized antennae, and of bees trained with free antennae and tested with immobilized antennae (groups $\mathrm{b}$ and c, respectively; see Materials and Methods). The bees did not show side-specific PE reaction times in any of the several tests if their antennae had been immobilized prior to training (Fig. 2A-E). And their reaction times did not differ from those of the untrained bees (Fig. 2A-E; Mann-Whitney test, test $1 \mathrm{~h}: P_{\text {ut vs. I }}=0.4, P_{\text {ut vs. D }}=$ 0.1 ; test $2 \mathrm{~h}: P_{\text {ut vs. I }}=0.5, P_{\text {ut vs. D }}=0.5$; test 3 h: $P_{\text {ut vs. I }}=0.3, P_{\text {ut vs. }}$ D $=0.4$; test $24 \mathrm{~h}: P_{\text {ut vs. I }}=0.3, P_{\text {ut vs. D }}=0.6$; and test $24 \mathrm{~h}_{(\text {we) }}: P_{\text {ut vs. }}$. $\left.\mathrm{I}=0.9, P_{\text {ut vs. }} \mathrm{D}=0.9\right)$. Similarly, they did not show side-specific PE reaction times when tested $1,2,3$, and $24 \mathrm{~h}$ after training if their antennae had been immobilized just before testing (Fig. 2F-I), and, as before, their behavior did not differ from that of the untrained bees (Fig. 2F-I; Mann-Whitney test, test $1 \mathrm{~h}: P_{\text {ut vs. I }}=$ $0.9, P_{\text {ut vs. D }}=0.9$; test $2 \mathrm{~h}: P_{\text {ut vs. I }}=0.7, P_{\text {ut vs. } \mathrm{D}}=0.6$; test $3 \mathrm{~h}: P_{\text {ut vs. }}$ I $=0.3, P_{\text {ut vs. D }}=0.1$; and test $\left.24 \mathrm{~h}: P_{\text {ut vs. I }}=0.7, P_{\text {ut vs. } \mathrm{D}}=0.9\right)$. However, a tendency $(P=0.05)$ suggests that those bees tested only once $24 \mathrm{~h}$ after training did show side differences in their reaction times (Fig. 2J). Moreover, their PE reaction times following stimulation of the decreasing antenna were significantly higher than those of the untrained bees (Mann-Whitney test, test $24 \mathrm{~h}_{(\mathrm{we})}$ :

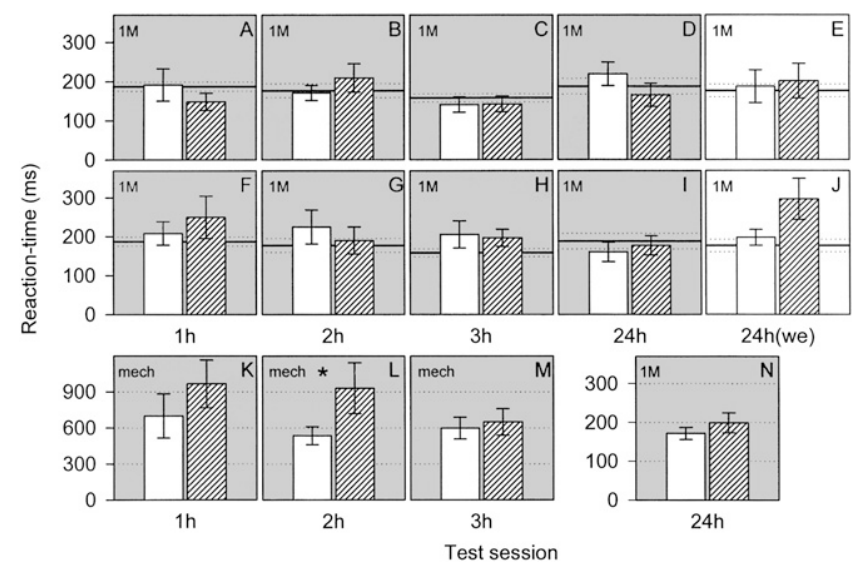

Figure 2. PE reaction times (mean $\pm S E M$, in milliseconds) following stimulation of the increasing and the decreasing antennae (white and dashed bars, respectively). Data are shown for the different tests. ( $A-E)$ Bees with immobilized antennae in both training and testing, and tested with $1 \mathrm{M}$ sucrose solution. (F- $/$ ) Bees trained with free antennae and tested with immobilized antennae; they were tested with $1 \mathrm{M}$ sucrose solution. $(K-N)$ Bees trained and tested with free antennae; they were tested 1, 2, and $3 \mathrm{~h}$ after training using mechanical stimulation (a dry toothpick) and $24 \mathrm{~h}$ after training using $1 \mathrm{M}$ sucrose solution. All groups were trained with $1 \mathrm{M}$ sucrose solution. In $A-J$, reference lines correspond to the reaction time of untrained bees tested simultaneously (mean \pm SEM, solid and dotted lines, respectively). Asterisks indicate statistical differences (Wilcoxon signed-rank test) between the reaction times which followed stimulation of the increasing and decreasing antennae: $(A) W=$ 37, $P=0.2, n=20 ;(B) W=-40, P=0.4, n=20 ;(C) W=-10, P=0.8, n=$ 20; $(D) W=33, P=0.1, n=13 ;(E) W=-5, P=0.8, n=14 ;(F) W=0$, $P=0.9, n=28 ;(G) W=34, P=0.6, n=26 ;(H) W=1, P=0.9, n=26 ;(I)$ $W=-19, P=0.4, n=10 ;(J) W=-117, P=0.05, n=23 ;(K) W=-22, P=$ $0.1, n=8 ;(L) W=-57, P=0.04, n=13 ;(M) W=-9, P=0.7, n=10 ;$ and (N) $W=-82, P=0.5, n=36$.
$\left.P_{\text {ut vs. I }}=0.3, P_{\text {ut vs. D }}=0.02\right)$. In addition, the bees trained with immobilized and free antennae showed similar reaction times during training (two-way repeated measures: effect $f_{\text {fixed }}$ vs. free, $F_{(1,160)}=2.9, P=0.09$; effect $t_{\text {training trials, }} F_{(5,800)}=20.7, P<$ 0.0001 ; and effect $\left.t_{\text {interaction, }} F_{(5,800)}=0.29, P=0.9\right)$. Overall, mechanosensory input from the proximal antennal joint played a key role in the formation and triggering of side differences in reaction time. We did not observe short- and long-term side-specific reaction times when mechanosensory input was hampered in both training and testing. Yet, hampering such input only during testing did not prevent us from recording long-term side differences in reaction time, indicating that gustatory input was sufficient to trigger long-term side-specific responses.

We also asked whether mechanosensory input is sufficient to trigger side-specific PE reaction times, and how repetitive mechanosensory input affects long-term side differences in reaction time. To answer these questions, we trained bees as before and tested them 1, 2, and $3 \mathrm{~h}$ after training with mechanical stimulation only, as well as with $1 \mathrm{M}$ sucrose solution $24 \mathrm{~h}$ after training (group d, see Materials and Methods). Following mechanical stimulation, we did not find side differences in reaction times 1 and $3 \mathrm{~h}$ after training (Fig. $2 \mathrm{~K}, \mathrm{M}$ ), whereas the reaction time of the increasing antenna was shorter than that of the decreasing antenna $2 \mathrm{~h}$ after training (Fig. 2L). We did not find side differences in reaction time when the same bees were tested with $1 \mathrm{M}$ sucrose solution $24 \mathrm{~h}$ after training (Fig. $2 \mathrm{~N}$ ). The bees' reaction time following mechanical stimulation of the antennae was approximately fourfold higher than those that followed sucrose stimulation of the antennae. The percentage of bees that extended the proboscis after mechanical stimulation of both antennae was 12\%-19\% (Fig. 4A-F, see below). Thus, mechanical input alone proved to be sufficient to evoke side-specific PE reaction times. Moreover, the bees that experienced mechanical stimulation 1, 2, and $3 \mathrm{~h}$ after training did not exhibit side differences in reaction time when tested with sucrose solution $24 \mathrm{~h}$ after training. This happened irrespective of whether or not the bees had responded to mechanical stimulation 1,2 , and $3 \mathrm{~h}$ after training. Therefore, long-term side differences in reaction time (Fig. 1E) were prevented by previous repetitive mechanical input.

\section{Specificity of the gustatory input}

We also asked whether side differences in PE reaction time can be triggered by gustatory inputs different than that of training. We trained the bees as before with $1 \mathrm{M}$ sucrose solution, and tested them with either $0.2 \mathrm{M}$ sucrose solution or water (groups e and $\mathrm{f}$, respectively, see Materials and Methods). We did not find side differences in reaction time after stimulation with either $0.2 \mathrm{M}$ sucrose (Fig. 3A-E) or water (Fig. 3F-J). This means that side differences in reaction time are specific to the gustatory input used during training, at least for stimuli weaker than the trained one, i.e., for decreasing sugar concentrations.

\section{Side-specific adjustments of the PE probability and duration}

In addition to the PE reaction time, we measured the probability and the duration of the bees' PER. For all the different groups of bees, we did not find side differences in the probability and the duration of PER in any of the tests (Supplemental Table S1).

\section{The reaction time, probability, and duration of a bee's PER}

Irrespective of the input side, we asked whether the reaction time, probability, and duration of a bee's PER depend on the stimulus used during testing, and whether these three variables change after training. To answer these questions, we first defined: (1) the mean 


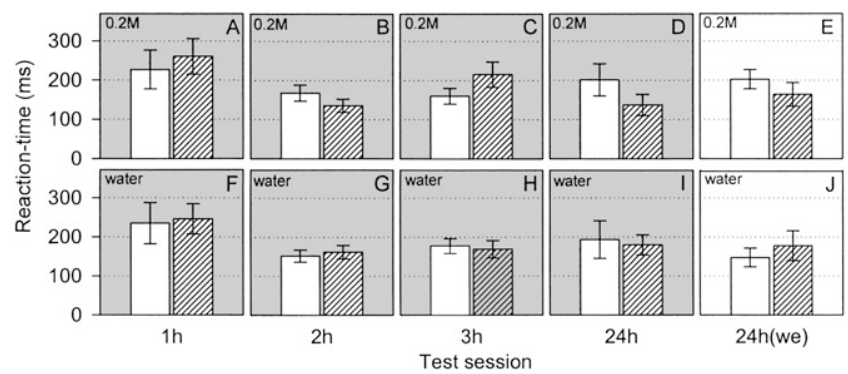

Figure 3. PE reaction times (mean \pm SEM, in milliseconds) following stimulation of the increasing and the decreasing antennae (white and dashed bars, respectively) with either $0.2 \mathrm{M}$ sucrose solution $(A-E)$ or water $(F-J)$. Data are shown for the different tests. Bees were trained using $1 \mathrm{M}$ sucrose solution. We found no difference (Wilcoxon signed-rank test) in the bees' reaction times following stimulation of either the increasing or decreasing antennae: $(A) W=-34, P=0.7, n=32 ;(B) W=-39, P=0.7$, $n=28 ;(C) W=60, P=0.5, n=25 ;(D) W=-14, P=0.7, n=12 ;(E) W=$ $-7, P=0.8, n=32 ;(F) W=-165, P=0.06, n=30 ;(G) W=95, P=0.2, n=$ $31 ;(H) W=99, P=0.2, n=29 ;(I) W=16, P=0.6, n=15$; and $(J) W=23$, $P=0.5, n=15$.

PE reaction time, as the average of the values from both antennae; (2) the PE probability, as the proportion of bees showing PER after stimulation of both antennae, calculated from the total number of tested bees; and (3) the mean PE duration, as the average of the values from both antennae. We used data from the naïve and trained bees tested with either 1 or $0.2 \mathrm{M}$ sucrose solution, water, or mechanical stimulation (groups a, e, f, and d, respectively), and from the untrained bees tested with $1 \mathrm{M}$ sucrose solution. Before testing, the naïve bees were neither trained nor fed. By contrast, the untrained bees were fed before testing (see Materials and Methods).

To answer whether the reaction time, probability, and duration of a bee's PER depend on the stimuli used during testing, we compared these variables across stimuli, i.e., as recorded from the bee's PER to the different stimuli, in both naïve and trained bees. We did not find differences in the mean PE reaction time after stimulation with 1 and $0.2 \mathrm{M}$ sucrose solution and water (Fig. 4A-F), and the corresponding values were lower than that recorded after mechanical stimulation of the antennae (Fig. 4A-F). The PE probabilities and durations were maximal after stimulation with $1 \mathrm{M}$ sucrose stimulation, intermediate with $0.2 \mathrm{M}$ sucrose solution and water, and minimal with mechanical stimulation alone (Fig. 4G-R). Thus, these three measures of a honeybee's PER depended on the stimuli used during testing.

Next, to answer whether the reaction time, probability, and duration of a bee's PER change after training, for each stimulus, we compared the performance of naïve and trained bees. We found that, irrespective of the stimuli used during testing, training induced changes in these three variables. In all the tests, the mean $\mathrm{PE}$ reaction time of the trained bees after stimulation with either 1 or $0.2 \mathrm{M}$ sucrose solution or water was lower than that of the naïve bees (Fig. 4A-F; Table 1A). The mean PE reaction time after mechanical stimulation appeared to not have changed after training, although reduced samples did not allow statistical comparisons. The PE probability following stimulation with $1 \mathrm{M}$ sucrose solution was similar in the naïve and trained bees in the tests performed 2 and $24 \mathrm{~h}$ after training, and significantly higher in the trained bees in the tests performed 1,3 , and $24 \mathrm{~h}_{\text {(we) }}$ after training (Fig. 4A-F; Table 1B). The PE probability following stimulation with $0.2 \mathrm{M}$ sucrose solution, water, or mechanosensory input was higher in the trained than in the naïve bees in all the different tests (Fig. 4A-F; Table 1B). Also, the mean PE duration following stimulation with either 1 or $0.2 \mathrm{M}$ sucrose solution or water was higher in the trained bees than in the naïve bees in all the different tests (Fig. 4G-L; Table 1C). We found a similar tendency while comparing the data from the trained and naïve bees which were mechanically stimulated, although reduced samples prevented statistics. Finally, we further examined the effect of training by comparing the performance of untrained and trained bees tested with $1 \mathrm{M}$ sucrose solution. The mean PE reaction time and the PE probability did not differ between the untrained and trained bees in all the different tests (Fig. 4B-L reference lines vs. first bar, Table 1A,B). The mean PE duration was significantly lower in the untrained bees than in the trained bees in all the different tests (Fig. 4M-R, reference lines vs. first bar, Table 1C). Thus, we found that (1) the PE probabilities and reaction times of the trained and the untrained bees did not differ from each other and (2) that the trained bees showed higher PE probabilities and lower reaction times than the naïve bees. One wonders whether the differences in PE probability and reaction time between the naïve and the trained bees arise from the bees' level of satiety. But higher satiation levels lead to lower PE

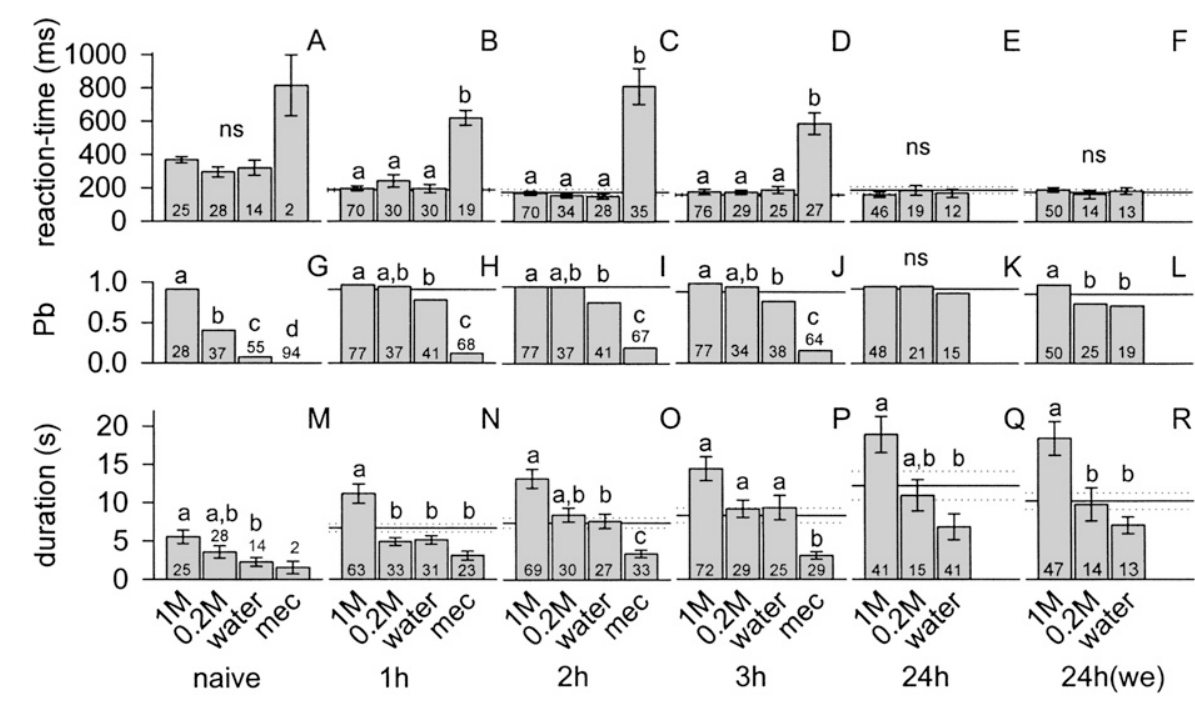

Figure 4. $(A-F)$ Mean PE reaction time (mean $\pm S E M$, in milliseconds), as the average of the values from both antennae. (G-L) PE probability, as the proportion of bees, which showed PER after stimulation of both antennae, calculated from the total amount of tested bees. (M-R) Mean PE duration (mean \pm SEM, in seconds), as the average of the values from both antennae. Data are shown for naïve and trained bees (i.e., in tests 1, 2, 3, $24 \mathrm{~h}$, and $24 \mathrm{~h}_{(\text {we) }}$ after training) tested with 1 or $0.2 \mathrm{M}$ sucrose solution, water, or mechanical stimulation. Reference lines indicate the values from untrained bees (mean \pm SEM, solid and dotted lines, respectively). In all cases different letters indicate $P<0.05$ of pairwise comparisons across stimuli after Kruskal-Wallis tests (for PE reaction time and durations) and/or G-tests (for PE probability): $(A) H_{3}=0.2, P=0.9 ;(B) H_{5}=42.7, P<0.0001 ;(C) H_{5}=61.8, P<0.0001 ;(D)$ $H_{5}=52.4, P<0.0001 ;(E) H_{4}=1.5, P=0.5 ;(F) H_{4}=0.7, P=0.7 ;(G) G_{3}=530.9, P<0.0001 ;(H) G_{3}=$ 185.4, $P<0.0001 ;(I) G_{3}=13.1, P=0.001 ;(J) G_{3}=20.1, P<0.0001 ;(K) G_{2}=1.3, P=0.5 ;(L) G_{2}=24.2$, $P<0.0001 ;(M) H_{3}=10.14, P=0.006 ;(N) H_{4}=47.1, P<0.0001 ;(O) H_{4}=52.8, P<0.0001 ;(P) H_{4}=$ 45.5, $P<0.0001 ;(Q) H_{3}=13.0, P=0.001$; and $(R) H_{3}=13.7, P=0.001$. Number of bees is given within the bars. Statistics for the comparisons between naïve and trained bees for the different stimuli, and between untrained (reference lines) and trained bees tested with $1 \mathrm{M}$ sucrose solution are shown in Table 1. 
Table 1. Statistics for the comparison between naïve and trained bees tested with the different stimuli, and between untrained and trained bees tested with $1 \mathrm{M}$ sucrose solution

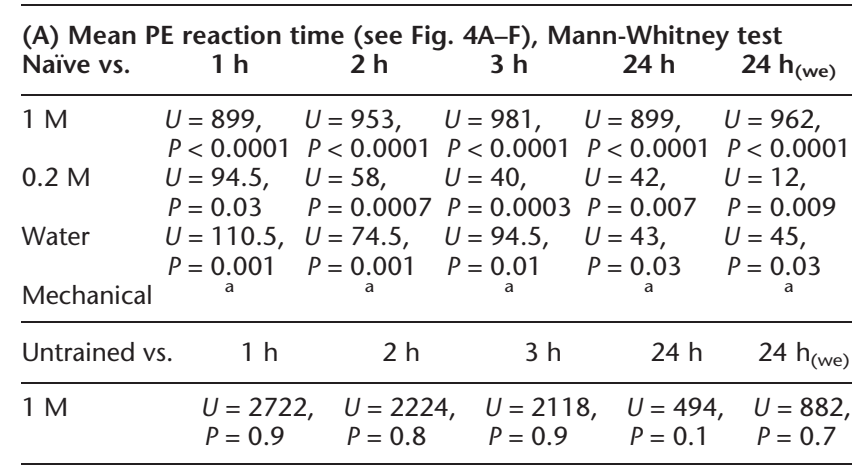

(B) PE probability (Fig. 4G-L), G-test

\begin{tabular}{|c|c|c|c|c|c|}
\hline Naïve vs. & $1 \mathrm{~h}$ & $2 \mathrm{~h}$ & $3 \mathrm{~h}$ & $24 \mathrm{~h}$ & $24 \mathrm{~h}_{\text {(we) }}$ \\
\hline $1 \mathrm{M}$ & $\begin{aligned} G & =6.75 \\
P & =0.03\end{aligned}$ & $\begin{aligned} G & =3.64, \\
P & =0.1\end{aligned}$ & $\begin{aligned} G & =13.5 \\
P & =0.002\end{aligned}$ & $\begin{array}{l}G=1.9 \\
P=0.4\end{array}$ & $\begin{aligned} G & =16.3 \\
P & =0.0003\end{aligned}$ \\
\hline & $\begin{array}{l}G=27.7 \\
P<0.0001\end{array}$ & $\begin{array}{l}G=27.7 \\
P<0.0001\end{array}$ & $\begin{array}{l}G=25.7 \\
P<0.0001\end{array}$ & $\begin{array}{l}G=19.9 \\
P<0.0001\end{array}$ & $\begin{array}{l}G=7.85 \\
P=0.005\end{array}$ \\
\hline & $\begin{array}{l}G=55.2 \\
P<0.0001\end{array}$ & $\begin{array}{l}G=51.7 \\
P<0.0001\end{array}$ & $\begin{array}{l}G=50.7 \\
P<0.0001\end{array}$ & $\begin{array}{l}G=37.2 \\
P<0.0001\end{array}$ & $\begin{array}{l}G=36.1 \\
P<0.0001\end{array}$ \\
\hline Mechanical & $\begin{array}{l}G=14.5 \\
P<0.0001\end{array}$ & $\begin{array}{l}G=24.4 \\
P<0.0001\end{array}$ & $\begin{array}{l}G=19.1 \\
P<0.0001\end{array}$ & & \\
\hline Untrained vs & $1 \mathrm{~h}$ & $2 \mathrm{~h}$ & $3 \mathrm{~h}$ & $24 \mathrm{~h}$ & $24 \mathrm{~h}_{\text {(we) }}$ \\
\hline $1 \mathrm{M}$ & $\begin{array}{l}G=3.6 \\
P=0.6\end{array}$ & $\begin{array}{l}G=0.08 \\
P=1\end{array}$ & $\begin{array}{l}G=10.7 \\
P=0.06\end{array}$ & $\begin{array}{l}G=0.4 \\
P=1\end{array}$ & $\begin{array}{l}G=8.2, \\
P=0.1\end{array}$ \\
\hline
\end{tabular}

(C) Mean PE duration (Fig. 4M-R), Mann-Whitney test

\begin{tabular}{|c|c|c|c|c|c|}
\hline Naïve vs. & $1 \mathrm{~h}$ & $2 \mathrm{~h}$ & $3 \mathrm{~h}$ & $24 \mathrm{~h}$ & $24 \mathrm{~h}_{\text {(we) }}$ \\
\hline $\begin{array}{l}1 \mathrm{M} \\
0.2 \mathrm{M} \\
\text { Water } \\
\text { Mechanical }\end{array}$ & $\begin{array}{l}U=428 \\
P=0.0005 \\
U=250 \\
P=0.005 \\
U=104 \\
P=0.006\end{array}$ & $\begin{array}{l}U=326 \\
P<0.0001 \\
U=126 \\
P<0.0001 \\
U=52 \\
P=0.0002 \\
\text { a }\end{array}$ & $\begin{array}{l}U=392 \\
P<0.0001 \\
U=112 \\
P<0.0001 \\
U=425 \\
P=0.0001\end{array}$ & $\begin{array}{l}U=142 \\
P<0.0001 \\
U=67 \\
P=0.0003 \\
U=37 \\
P=0.01\end{array}$ & $\begin{array}{l}U=159 \\
P<0.0001 \\
U=52 \\
P=0.0001 \\
U=28 \\
P=0.0002 \\
a\end{array}$ \\
\hline Untrained vs. & $1 \mathrm{~h}$ & $2 \mathrm{~h}$ & $3 \mathrm{~h}$ & $24 \mathrm{~h}$ & $24 \mathrm{~h}_{(\text {we) }}$ \\
\hline & $\begin{array}{l}U=163 \\
P<0.0\end{array}$ & $\begin{array}{ll}, \quad U=114 \\
01 & P<0.0\end{array}$ & $\begin{array}{ll}0, & U=12 \\
01 & P=0 .\end{array}$ & $\begin{array}{l}4, \quad U=355, \\
02 \quad P=0.02\end{array}$ & $\begin{array}{l}U=540 \\
P=0.003\end{array}$ \\
\hline
\end{tabular}

The corresponding mean $( \pm$ SEM) values are presented in Figure 4 . ${ }^{a}$ Reduced samples of naïve bees did not allow statistical comparisons. ${ }^{\mathrm{b}} \mathrm{No}$ data were collected at $24 \mathrm{~h}$.

probabilities. (In fact, satiated bees do not extend their proboscises reflexively in response to sugar stimulation.) Moreover, evidence shows that the level of satiation does not correlate to the PE reaction time (Gil et al. 2008). Hence, the differences that we found in the PE probability and reaction times of the naïve and the trained bees cannot be accounted for by their respective satiation levels.

\section{Discussion}

We report a hitherto unknown form of side-specific learning in honeybees. Our results show that honeybees learn to associate the gustatory and mechanical stimulation of each antenna with either increasing or decreasing reward magnitudes. After side-specific training, the bees extended their proboscises earlier after stimulation of the antenna that had been linked to increasing volumes of sugar solution than after stimulation of the antenna that had been linked to decreasing volumes of sugar solution (Fig. 1A-E). It follows that a built-in change detector allows honeybees to compute differences in reward magnitude across feeding events, and that such computations can be side specific. As a result, estimates of expected magnitudes of a given reward can be linked to specific input of each antenna. We shall refer to such estimates as "side-specific reward memories." These memories underlie a form of side-specific learning based on a measurable property of the experienced reward, namely, that its magnitude increases or decreases over time. Their activation leads to both short- and longterm side differences in the bees' PE reaction time (Fig. 1A$\mathrm{C}, \mathrm{E})$. The ensuing long-term side-specific responses can be prevented by stimulating the bees' antennae repeatedly after training (Fig. 1D), which reveals an extinction effect on the side-specific association. Short-term side-specific responses arise from the joint effects of the increasing and decreasing reward schedules (Fig. 1A), whereas long-term side-specific responses arise from the effect of the decreasing reward schedule alone (Fig. 1E). In our experiments, we did not find side differences in the bees' reaction time during training (Fig. 1F,G), which suggests that integration over the six training trials and a subsequent consolidation period are necessary for side-specific responses to be evoked.

The wonder arises as to whether the side-specificity of such memories derives from an association between the mechanical input (from the mechanoreceptors located between the scapes and the head) that the bees had experienced during training and the expected magnitudes of a given reward. This view is consistent with the fact that (1) short- and long-term side-specific responses were not observed when mechanosensory input was absent during both training and testing (Fig. 2A-E); (2) mechanosensory input alone was sufficient to evoke and to extinguish side-specific responses (Fig. 2L,N); and (3) short-term side-specific responses were not observed when mechanosensory input was absent during testing (Fig. 2F-I). In the latter situation, however, the gustatory input alone evoked long-term side-specific responses (Fig. 2J). Taken together, the results described above suggest that mechanical and gustatory inputs interact in the development and subsequent retrieval of side-specific reward memories, and that they do so in such a way that the gustatory input acquires the capacity to retrieve these memories only in the long term. Further experiments are required to ponder the relative involvement of mechanical and gustatory inputs in the development of side specificity. Interestingly, the bees did not show side differences in their reaction time when tested with a gustatory stimulus weaker than that of training (Fig. 3). This suggests that side differences are largely specific regarding the nature of the gustatory input. Whether this type of specificity is also observed when the test stimulus is stronger than that of training remains an open question, however.

In addition to the side differences in the bees' $\mathrm{PE}$ reaction time, we found that training also exerts an overall effect on the reaction time, the probability and duration of the bees' PER to different stimuli (Fig. 4; Supplemental Fig. S2; see also Results). Such an overall effect becomes evident if one compares the average of both antennae data for each of such three measures between trained and naïve bees. Training decreased the average reaction time and increased the probability and duration of the bees' PER. This effect was invariant to the test stimulus, with the exception of the reaction time following mechanical stimulation of the antennae that did not change after training (Fig. 4; Supplemental Fig. S2). The increase in PE probability suggests that stimulation of the antenna, which involves gustatory and mechanical inputs, serves not only as an unconditioned stimulus (US), but also as a conditioned stimulus (CS) for subsequent rewards. This interpretation is consistent with previous reports indicating that a honeybee's PER can be conditioned to mechanical stimulation of the antenna (Menzel et al. 2001; Giurfa and Malun 2004), and to the water vapor emanating from a drop of sucrose solution (Kuwabara 1957). 
Our results also document how the regulation of the probability, the reaction time, and the duration of a honeybee's PE involves not only a series of common interacting elements, but also separate ones, which are specific for each of these three measures. We found training-related changes in the overall probability, reaction time, and duration of the bees' PERs, which did not necessarily correlate with each other (Fig. 4; Supplemental Fig. S2). We also found side differences in the reaction time but not in the probability or the duration of the bees' PER (Fig. 1; Supplemental Table S1). Further, we found that side differences in reaction time were specific to the test stimulus (Fig. 3) and independent of the overall probability of the bees' PER. Thus, the tests with either 1 or $0.2 \mathrm{M}$ sucrose solution or water gave similar PER probabilities, but only those with $1 \mathrm{M}$ sucrose solution led to side differences in reaction time. Moreover, mechanical stimulation led to both very low PE probabilities and side differences in reaction time (Fig. 4; Supplemental Fig. S2). These results are consistent with the idea that the side differences in reaction time depend upon the activation of side-specific reward memories formed on the basis of a specific property of the offered reward, namely, that its magnitude changed over time. They are also consistent with the idea that such memories are formed in parallel to those arising from a contingency between the stimulation of the antennae (as a CS) and the offered reward (as a US). This is important because it indicates that PE reaction time can be a measure of a honeybee's anticipatory response to specific rewards. The adjustment of a subject's anticipatory response to reward is typically thought of as being rooted in the subject's expectations of reward (Schultz 2006). Along the same line, one might conclude from our observations that honeybees learn to "expect" at least two different reward magnitudes.

In a previous study, we found that free-flying honeybees adjust their persistence to search for food based on a specific property of a previously experienced reward, namely, that its magnitude increased over time (Gil et al. 2007). Next, we found that harnessed honeybees adjust their PE reaction times based on the same specific property of reward (Gil et al. 2008). Here, we report that the latter adjustment can also be side specific. Thus, we have developed a laboratory procedure suitable to examine behavioral correlates of memories on specific reward properties, which includes within-animal controls. This is important because honeybees assign sugar solution with subjective values of reward (Page et al. 1998; Scheiner et al. 2005). Furthermore, because the $\mathrm{PE}$ reaction time correlates well with the reaction time of muscles involved in the movements of a honeybee's proboscis (Fig. 1H), our preparation also includes a physiological correlate of behavior. The substitution of a behavioral response like the honeybee PER by such a physiological measure may be an important contribution to future studies using pharmacological, electrophysiological, and optophysiological techniques. Such studies would focus on brain areas, where projections of gustatory receptors from the antennae and proboscis, and mechanosensory receptors from the antennae converge. Evidence points toward the dorsal lobe and the suboesophageal ganglion as neuropils where the processing of both mechanosensory input from the antennae and gustatory input from both the antennae and the proboscis actually occurs (Suzuki 1975; Maronde 1991; Haupt 2005). Our results may guide future anatomical and physiological studies aiming to characterize the neural correlates of memories on specific reward properties.

\section{Materials and Methods}

This work complies with the current laws regarding experiments with insects. We caught honeybees (Apis mellifera carnica) at a hive's entrance, and harnessed them in metal tubes by strips of tape between their head and thorax, so that they could freely move their antennae and mouth parts (Bitterman et al. 1983). After harnessing, we placed the bees in racks, fed them with $10 \mu \mathrm{L}$ of unscented $1.2 \mathrm{M}$ sucrose solution, and kept them overnight in a dark humidified chamber. Next, we presented the bees with six training trials on the following morning. The term "training trial" refers to the stimulation of a bee's antenna with a toothpick soaked in sucrose solution, and the subsequent presentation of a given volume of sucrose solution delivered to the animal's proboscis. Each training trial lasted $\sim 30 \mathrm{sec}$. First, we moved a bee from a rack to the training site. Following a $10 \mathrm{sec}$ accommodation period, we stimulated one of its antennae for $2 \mathrm{sec}$ by touching it with a toothpick soaked in an unscented $1 \mathrm{M}$ sucrose solution, and then fed the animal for $10 \mathrm{sec}$ with a given volume of the same sucrose solution delivered to its proboscis by means of a micrometer syringe. The bee remained in the training site for 8 sec after feeding, and was then placed back in the rack. We performed a side-specific training in which the stimulation of each antenna was coupled with either increasing (small: $0.4 \mu \mathrm{L}$; medium: $1 \mu \mathrm{L}$; and large: $1.6 \mu \mathrm{L}$ ) or decreasing (large: $1.6 \mu \mathrm{L}$; medium: $1 \mu \mathrm{L}$; and small: $0.4 \mu \mathrm{L}$ ) volumes of sucrose solution throughout six consecutive training trials. The intertrial interval was $10 \mathrm{~min}$. The total volume of sucrose solution that each bee received throughout the entire training session was $6 \mu \mathrm{L}$. A bee's antennae were stimulated alternately, so that one antenna, either left or right, was stimulated in the first, third, and fifth training trial, whereas the other antenna was stimulated in the second, fourth, and sixth training trial. Thus, for example, when the right and the left antennae were assigned to the increasing and the decreasing reward schedules, respectively_in which case we refer to the increasing and the decreasing antennae, respectively-the training procedure was as follows: In the first training trial, we stimulated the right antenna and fed the bee with $0.4 \mu \mathrm{L}$ of sucrose solution; in the second training trial, we stimulated the left antenna and fed the bee with $1.6 \mu \mathrm{L}$ of sucrose solution; in the third and fourth training trials, we stimulated the right and left antennae, respectively, and fed the bee with $1 \mu \mathrm{L}$ of sucrose solution in both trials; in the fifth training trial, we stimulated the right antenna and fed the bee with $1.6 \mu \mathrm{L}$ of sucrose solution; and, finally, in the sixth training trial, we stimulated the left antenna and fed the bee with $0.4 \mu \mathrm{L}$ of sucrose solution. Half of the bees were presented with an increasing reward schedule following stimulation of the right antenna, whereas the other half with an increasing schedule following stimulation of the left antenna. Also, half of the bees were trained by starting with the small sugar solution volume (increasing reward schedule), and half by starting with the large sugar solution volume (decreasing reward schedule).

In addition, the trained bees were divided into two groups. One group was tested $1,2,3$, and $24 \mathrm{~h}$ after training, while the other group was tested only $24 \mathrm{~h}$ after training (henceforth, test 24

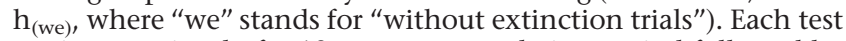
session consisted of a $10 \mathrm{sec}$ accommodation period followed by a 2 sec stimulation of the antenna. Next, the bee remained in the testing site for $8 \mathrm{sec}$, and was then placed back in the rack. After 20 $\mathrm{min}$, the test procedure was repeated with the other antenna. Half of the bees were tested by stimulating first the right and then the left antenna. The other half was tested in the opposite way. Depending on the experimental group (see below), the 2 sec stimulation of each antenna was performed with a toothpick soaked in either $1 \mathrm{M}$ sucrose solution, $0.2 \mathrm{M}$ sucrose solution, or water, or with a dry toothpick. The evening following the training trials and the test sessions made 1,2 , and $3 \mathrm{~h}$ after training, the bees were fed with $5 \mu \mathrm{L}$ of unscented $1.2 \mathrm{M}$ sucrose solution and kept overnight inside a dark humidified chamber. To feed the bees after harnessing and training, we released their PERs by stimulating their proboscis with sucrose solution, instead of their antennae, thereby avoiding triggering their PERs in a way similar to that of the training trials and tests.

We evaluated the performance of six groups of bees trained as described above. The bees of these groups differed in two ways. First, they could be tested with different stimuli, namely, 1 $\mathrm{M}$ sucrose, $0.2 \mathrm{M}$ sucrose, water, or mechanical stimulation. Second, they could have their antennae immobilized or not. 
Immobilization of the antennae was performed $30 \mathrm{~min}$ prior to either training or testing, depending on the treatment (see below). To immobilize the bees' antennae, we fixed both antennal scapes (the basal segment of the antennae) to the head using acrylic paint, so that the animals could freely move only the flagellum of each antenna. Thus, the six groups were: (1) bees that had their antennae free in both training and testing, and that were tested with $1 \mathrm{M}$ sucrose solution; (2) bees that had their antennae immobilized in both training and testing, and that were tested with $1 \mathrm{M}$ sucrose solution; (3) bees that had their antennae free in training and immobilized in testing, and that were tested with $1 \mathrm{M}$ sucrose; (4) bees that had their antennae free in both training and testing, and that were tested 1,2 , and $3 \mathrm{~h}$ after training with mechanical stimulation, as well as $24 \mathrm{~h}$ after training with $1 \mathrm{M}$ sucrose solution; (5) bees that had their antennae free in both training and testing, and were tested with $0.2 \mathrm{M}$ sucrose solution; and (6) bees that had their antennae free in both training and testing, and were tested with water. Additionally, two groups of untrained bees were fed with $6 \mu \mathrm{L}$ of unscented $1 \mathrm{M}$ sucrose solution, and subsequently tested with $1 \mathrm{M}$ sucrose solution. One of such group was tested $1,2,3$, and $24 \mathrm{~h}$ after feeding, whereas the other group was tested only $24 \mathrm{~h}$ after feeding (test $24 \mathrm{~h}_{\text {(we) }}$ ). We also evaluated the PER to $1 \mathrm{M}$ sucrose, $0.2 \mathrm{M}$ sucrose, water, and mechanical stimulation of the antennae of bees that were only harnessed, fed with $10 \mu \mathrm{l}$ of $1.2 \mathrm{M}$ sucrose solution, and kept overnight in a dark humidified chamber. We shall refer to these bees as "naïve bees." Up to four groups per day were run in parallel and assayed in a semirandom way.

We video-recorded the bees' PERs at 60 frames $^{\prime} \mathrm{sec}^{-1}$ during the training trials and tests, and subsequently analyzed the videos frame by frame. The bees that did not respond to sucrose stimulation during training were excluded from the analysis. We characterized the bees' PER to antennal stimulation using four variables. The first of such variables (1) was the PE reaction time (milliseconds), defined as the time elapsed between the onset of antennal stimulation and the first movement of a bee's proboscis, provided that such movement subsequently led to a successful extension of the bee's proboscis (see below). In each test session, we obtained two reaction-time values per bee, those following stimulation of the bee's "increasing" and "decreasing" antennae. For the sake of comparison, we calculated a "differential reaction time," as the difference between the reaction time following stimulation of the decreasing and the increasing antennae $(D-$ $I$ ). Next, for the sake of normalization, we divided such difference by the highest reaction time obtained for each bee in each test ([D-I]/D, if $D>I$, or $[D-I] / I$, if $I>D)$, which allowed us to express group differences in percentage. The second (2) variable was the PE probability, defined as the proportion of bees that successfully extended their proboscises, as calculated from the total number of bees involved in each test. A successful extension was scored as such if the proboscis crossed an imaginary line between the tips of a bee's opened mandibles. Because each animal was tested twice in each test session, we calculated three different PE probabilities, defined as the proportions of animals that responded to the stimulation of the increasing antenna, the stimulation of the decreasing antenna, and the stimulation of both antennae, as calculated from the total number of bees involved in each test. The third (3) variable was the PE duration, defined as the total amount of time during which the bees remained with the proboscis extended within a $60 \mathrm{sec}$ time period following antennal stimulation. In parallel to the video recordings, we made electromyogram recordings (EMGs) of the bees' M17 muscles (Rehder 1987), a pair of bilaterally symmetrical muscles involved in the extension of the proboscis (Snodgrass 1956). We made two tiny holes at the level of the lateral ocellus near the dorsal rim of each compound eye, and inserted a metal wire $(0.125 \mathrm{~mm}$ diameter silver wire) $1-2 \mathrm{~mm}$ into each of these holes to record from both M17 muscles. The reference electrode was inserted in one compound eye (Rehder 1987). Recordings were made using a CED micro-1401 interface and Spike2 software (Cambridge Electronic Design). In this way, following antennal stimulation we obtained EMGs of both the ipsi- and the contralateral M17 muscles. Thus, we calculated a fourth (4) variable, namely, the M17 reaction time (in ms, either ipsi- or contralateral), as the time elapsed between the onset of the antennal stimulation and the first spike of each muscle.

Data were analyzed by means of one sample $t$-test, Wilcoxon signed-rank test, two-way repeated-measures ANOVA and Tukey's multiple comparisons, Mann-Whitney test, $t$-test, Spearman correlation, and $G$-test. Nonparametric tests were used when data did not fulfill the requirements of parametric tests.

\section{Acknowledgments}

M.G. and R.J.D.M. are indebted to Dr. J.A. Núñez (University of Buenos Aires) for fruitful discussions. This work was supported by the Deutsche Forschungsgemeinschaft (The German Research Council, grant to R.M.).

\section{References}

Bitterman ME, Menzel R, Fietz A, Schäfer S. 1983. Classical conditioning of proboscis extension in honeybees (Apis mellifera). J Comp Psychol 97: $107-119$.

Braun G, Bicker G. 1992. Habituation of an appetitive reflex in the honeybee. J Neurophysiol 67: 588-598.

Gil M, De Marco RJ, Menzel R. 2007. Learning reward expectations in honeybees. Learn Mem 14: 491-496.

Gil M, Menzel R, De Marco RJ. 2008. Does an insect's unconditioned response to sucrose reveal expectations of reward? PLoS One 3: e2810. doi: 10.1371/journal.pone.0002810.

Giurfa M, Malun D. 2004. Associative mechanosensory conditioning of the proboscis extension reflex in honeybees. Learn Mem 11: 294-302.

Haupt SS. 2004. Antennal sucrose perception in the honey bee (Apis mellifera L.): Behaviour and electrophysiology. J Comp Physiol [A] 190: $735-745$.

Haupt SS. 2005. Das gustatorische system und antennales lernen in der honigbiene (Apis mellifera L.). PhD thesis, Technischen Universität Berlin, Germany.

Kuwabara M. 1957. Bildung des bedingten reflexes von Pavlovs typus bei der honigbiene, Apis mellifica. J Fac Hokkaido Univ Ser VI Zol 13: 458464 .

Macmillan CS, Mercer AR. 1987. An investigation of the role of dopamine in the antennal lobes of the honeybee, Apis mellifera. J Comp Physiol 160: $359-366$.

Markl H. 1971. Propioceptive gravity perception in Hymenoptera. In Gravity and the organism (eds. S Gordon, M Cohen), pp. 185-194. University of Chicago Press, Chicago, IL.

Maronde U. 1991. Common projection areas of antennal and visual pathways in the honeybee brain, Apis mellifera. J Comp Neurol 309: 328-340.

Menzel R, Manz G, Menzel RM, Greggers U. 2001. Massed and spaced learning in honeybees: The role of CS, US, the inter-trial interval and the test interval. Learn Mem 8: 198-208.

Page RE Jr, Erber J, Fondrk MK. 1998. The effect of genotype on response thresholds to sucrose and foraging behavior of honeybee (Apis mellifera L.). J Comp Physiol [A] 182: 489-500.

Rehder V. 1987. Quantification of the honeybee's proboscis reflex by electromyographic recordings. Ins Physiol 33: 501-507.

Sandoz J-C, Menzel R. 2001. Side-specificity of olfactory learning in the honeybee: Generalization between odors and sides. Learn Mem 8: 286-294.

Sandoz J-C, Hammer M, Menzel R. 2002. Side-specificity of olfactory learning in the honeybee: US input side. Learn Mem 9: 337-348.

Scheiner R, Kuritz-Kaiser A, Menzel R, Erber J. 2005. Sensory responsiveness and the effects of equal subjective rewards on tactile learning and memory of honeybees. Learn Mem 12: 626-635.

Schneider D. 1964. Insect antennae. Annu Rev Entomol 9: 103-122.

Schultz W. 2006. Behavioral theories and the neurophysiology of reward. Annu Rev Psychol 57: 87-115.

Smith BH, Menzel R. 1989. An analysis of variability in the feeding motor program of the honey bee; the role of learning in releasing a modal action pattern. Ethol 82: 68-81.

Snodgrass RE. 1956. Anatomy of the honey bee. Comstock, Ithaca, NY.

Suzuki H. 1975. Antennal movements induced by odour and central projection of the antennal neurons in the honeybee. J Insect Physiol 21: $831-847$.

Takeda K. 1961. Classical conditioned response in the honey bee. J Insect Physiol 6: 168-179.

Received March 17, 2009; accepted in revised form April 13, 2009. 


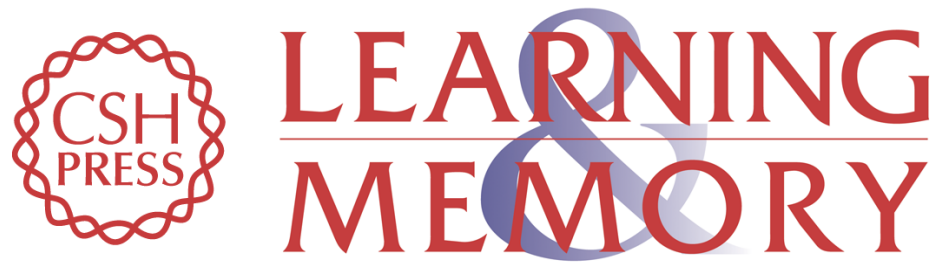

\section{Side-specific reward memories in honeybees}

Mariana Gil, Randolf Menzel and Rodrigo J. De Marco

Learn. Mem. 2009, 16:

Access the most recent version at doi:10.1101//m.1419109

Supplemental http://learnmem.cshlp.org/content/suppl/2009/06/29/16.7.426.DC1

References This article cites 20 articles, 6 of which can be accessed free at: http://learnmem.cshlp.org/content/16/7/426.full.html\#ref-list-1

License

Email Alerting Receive free email alerts when new articles cite this article - sign up in the box at the Service top right corner of the article or click here. 